\title{
Utilidad del monitor externo de eventos en el tratamiento del paciente con fibrilación auricular
}

\section{The usefulness of an extreme event monitor in the atrial fibrillation patient}

\author{
Guillermo Mora-Pabón ${ }^{\mathrm{a}, \mathrm{b}, \mathrm{c}, \mathrm{d}}$ \\ a Departamento de Medicina Interna, Universidad Nacional de Colombia, Bogotá, Colombia \\ b Fundación Santafé de Bogotá, Bogotá, Colombia \\ c Hospital Universitario Nacional, Bogotá, Colombia \\ 'Clínica San Rafael, Bogotá, Colombia
}

Recibido el 13 de octubre de 2016; aceptado el 22 de noviembre de 2016

Disponible en Internet el 5 de deciembre de 2016

\section{Introducción}

El diagnóstico de fibrilación auricular se fundamenta en hallazgos electrocardiográficos de ausencia de onda $P$ e irregularidad de los RR (irregularmente irregular). Inicialmente, la única herramienta diagnóstica era el electrocardiograma (ECG), que tiene un alto rendimiento en fibrilación auricular permanente, pero es bajo en la fibrilación auricular paroxística. Aunque la monitorización electrocardiográfica continua (Holter) ofrece mayor posibilidad de diagnóstico, existe un gran número de pacientes que tienen eventos con periodicidad mayor a 24 o 72 horas y constituyen un reto para lograr demostrar la presencia de fibrilación auricular, dadas las grandes consecuencias terapéuticas y pronósticas de esta enfermedad.

En este contexto, se desarrollaron los monitores externos de eventos, los cuales están conectados al paciente a través de electrodos y cuentan con batería de autonomía de cuatro semanas de duración. Pueden grabar eventos activados por el paciente (sintomáticos) y algunos hacen registros automáticos de bradicardia, taquicardia e irregularidad del RR (fibrilación auricular) sin necesidad de activación por el paciente (eventos asintomáticos) ${ }^{1}$. Su desventaja radica en

Correo electrónico: gmorap@unal.edu.co que detectan el inicio de la fibrilación auricular pero no el final del evento, así que no aportan información acerca de la carga arrítmica.

Existen también sistemas de telemetría ambulatoria y monitorización tipo parches, los cuales envían la información de los trazos almacenados a una central que categoriza los datos y remite la información filtrada para análisis del médico. A diferencia del monitor externo, estos dispositivos pueden almacenar registros hasta el final del evento, con lo que dan información de la carga anatómica².

\section{Diagnóstico de fibrilación auricular}

En el paciente con síntomas sugestivos de arritmias, la fibrilación auricular debe ser un diagnóstico a descartar. En las personas con palpitaciones, las guías del Colegio Colombiano de Electrofisiología y la Sociedad Colombiana de Cardiología consideran indicación clase । la utilización del monitor externo de eventos en aquellos sin factores de alto riesgo, con una intercurrencia de eventos menor a cuatro semanas ${ }^{1}$.

Sin embargo, el diagnóstico de fibrilación auricular en poblaciones con palpitaciones y bajo riesgo vascular, no es alto. Un estudio en población local, llevada a monitorización externa de eventos, evaluó 203 pacientes con edad promedio de 49 años (19,7\% eran mayores de 65 años). La indicación del estudio fue palpitaciones en 161 pacientes 
$(79,3 \%)$ y de ellos solo en $3(1,4 \%)$ se documentó fibrilación auricular ${ }^{3}$. Por otra parte, es importante resaltar que la sintomatología asociada a fibrilación auricular es muy variada e incluye debilidad, fatiga, disnea, poliuria y síncope, en menor frecuencia, de ahí que sea cardinal tener un alto grado de sospecha para buscar esta arritmia en pacientes con dichas características.

Una manifestación inicial de la fibrilación auricular es el desarrollo de eventos embólicos, comúnmente al sistema nervioso central; en ellos es importante el diagnóstico de la arritmia dada la necesidad de anticoagulación para prevenir nuevos episodios.

El riesgo de eventos cerebro-vasculares aumenta cinco veces en presencia de fibrilación auricular y se ha calculado que un $15 \%$ de eventos cerebro-vasculares se atribuye a esta arritmia ${ }^{4}$, los cuales son más fatales o discapacitantes que los no relacionados con ella. Se estima que 50 a $60 \%$ de estos son de origen arterioesclerótico y una minoría se relaciona con causas raras como vasculitis o estados hipercoagulables ${ }^{5}$. Después de la evaluación inicial cerca del $25 \%$ de infartos isquémicos semejantes a un evento embólico no tienen documentado el origen de éste. Estos eventos se clasifican como eventos cerebro-vasculares criptogénicos, o más recientemente como eventos cerebro-vasculares embólicos de origen no determinado ${ }^{7}$. Muchos de estos pacientes pueden tener fibrilación auricular no documentada ${ }^{8,9}$.

El diagnóstico de fibrilación auricular se ha basado tradicionalmente en la toma de ECG, monitorización electrocardiográfica continua intrahospitalaria y Holter en la hospitalización índice. Aunque hay varios estudios pequeños acerca de la utilidad del monitor externo de eventos en el diagnóstico de eventos cerebro-vasculares criptogénicos, recientemente fue publicado el $\mathrm{EMBRACE}^{8}$, en el que se incluyeron 572 pacientes mayores de 55 años sin antecedente de fibrilación auricular con eventos cerebrovasculares en los anteriores seis meses, sin una causa determinada luego de imágenes neurovasculares, ecocardiograma, ECG y Holter. Se asignaron en forma aleatoria a monitor externo de eventos, con detección automática de fibrilación auricular, por 30 días o un nuevo Holter. Se consideró un hallazgo positivo la detección de fibrilación auricular de duración mayor a 30 segundos. Estos eventos se detectaron en $16,1 \%$ en el grupo del monitor vs. $32 \%$ en el grupo control, con un número necesario a evaluar de 8 . Esta población era mayor (edad promedio $72,5+8$ años); más de una tercera parte correspondía a mayores de 75 años, de modo que no es claro si estos hallazgos se pueden extrapolar a poblaciones más jóvenes. Un pequeño estudio encontró que la positividad en pacientes menores a 60 años era solo del $2,7 \%^{10}$.

De otro lado, el uso de telemetría ambulatoria y monitores tipo parche, ha tenido un desarrollo importante en los últimos años. Una serie inicial de 56 pacientes con eventos cerebro-vasculares criptogénicos fueron evaluados con telemetría ambulatoria por 21 días encontrando fibrilación auricular en $23 \%$ de los casos; sin embargo $85 \%$ tenían duración menor a 30 segundos $^{6}$. Otro estudio que evaluó la duración de la telemetría ambulatoria para el diagnóstico de fibrilación auricular, encontró que el mejor tiempo de monitorización es de 21 días, obteniéndose 17,3\% de diagnóstico en la población estudiada con eventos cerebrovasculares o ataque isquémico transitorio criptogénico ${ }^{11}$.
Con este método diagnóstico otros autores hallaron una prevalencia de 14\% (IC 95\% 9-18\%) en 227 pacientes con eventos embólicos criptogénicos; además encontraron como predictores de riesgo de fibrilación auricular: edad mayor a 60 años o infarto cerebral cortical o cerebelar previo ${ }^{12}$. No obstante, un estudio reciente llamó la atención sobre la evaluación adecuada de estos pacientes; en 85 de ellos se utilizó comúnmente ecocardiograma transtorácico $(89,4 \%)$, transesofágico $(68,2 \%)$ y RNM completa $(38,8 \%)$ para aclarar la causa del evento cerebro-vascular. Con esta evaluación más completa solo hubo $4 \%$ de positividad para fibrilación auricular en la telemetría ambulatoria por 30 días $^{13}$.

Una revisión sistemática y metaanálisis ${ }^{14}$ recientemente publicado, evaluó la monitorización electrocardiográfica en pacientes con eventos cerebro-vasculares o ataque isquémico transitorio reciente. Dividieron la monitorización en cuatro fases: en la fase 1 (en sala de urgencias) se utilizó el ECG de ingreso, en la fase 2 (intrahospitalario) se emplearon ECG seriados, monitorización continua electrocardiográfica, telemetría y Holter, en la fase 3 (ambulatorio temprano) se empleó el Holter, y en la fase 4 (ambulatorio tardío) se manejó telemetría ambulatoria, monitor externo de asa y monitor implantable. Los autores analizaron 50 estudios con 11.658 pacientes que satisfacían sus criterios de inclusión. En la fase 1, el ECG diagnosticó fibrilación auricular en 7,7\% de pacientes (IC 95\% 5-10,8). En la fase 2 (intrahospitalario) se diagnosticó fibrilación auricular con ECG seriados en 5,6\% de pacientes (IC 95\% 3,6-7,9), con monitorización continua en $7 \%(3,9-10,8)$, telemetría en $4,1 \%(0,9-9,2)$ y Holter en $4,5 \%(2,7-6,7)$. No se encontraron diferencias entre estos métodos $(p=0,46)$. El rendimiento diagnóstico combinado fue del $5,1 \%(3,8-6,5)$. En la fase 3 (ambulatorio temprano) se incluía solo Holter de 1 a 7 días y la producción diagnóstica fue del 10,7\% (IC 95\% 5,6-17,2). En la fase 4 (ambulatoria tardía) no hubo diferencias entre la producción diagnóstica de la telemetría ambulatoria $(15,3 \%$ IC95\% 5,3-29,3), el monitor externo de eventos (16,2\% IC95\% 9,3-24,6) y el monitor implantable (16,9\% IC95\% 10,3-24,9). La proporción combinada de diagnóstico en fase 4 fue del $16,9 \%$ (IC95\% 13-21,2). El uso de las cuatro fases lleva a un diagnóstico de fibrilación auricular posteventos cerebrovasculares o ataque isquémico transitorio del $23,7 \%$ (IC95\% 17,2-31). Si el trabajo diagnóstico terminara en la fase 2 o 3, solo se haría diagnóstico en el 50,2 o el $81,9 \%$ de pacientes con fibrilación auricular, respectivamente. Al igual que muchos metaanálisis, existen limitaciones; por ejemplo algunos estudios no incluían el ataque isquémico transitorio, en otros sólo se incluía fibrilación auricular de más de 30 segundos de duración, los pacientes que llegaban a la fase 4 tenían procedimientos negativos previamente y se seguían considerando de alto riesgo, y finalmente la estratificación en cuatro fases fue subjetiva de los autores.

\section{Evaluación del tratamiento}

El monitor externo de eventos posterior a la ablación por fibrilación auricular, se ha utilizado en la evaluación de tipos de fibrilación auricular en el período de blanking, así como para determinar la causa de síntomas y detectar eventos asintomáticos de fibrilación auricular ${ }^{15}$. Un estudio que incluyó 72 pacientes postablación, utilizó un 
monitor con autodetección de fibrilación auricular por tres meses, mediante seguimiento del procedimiento. Aunque $65 \%$ tuvieron al menos un evento de fibrilación auricular, la ausencia de eventos en las primeras dos semanas tenía sensibilidad del $90 \%$ para ausencia de fibrilación auricular en seis meses ${ }^{16}$.

La ablación de fibrilación auricular con aislamiento de venas pulmonares disminuye a la mitad los eventos sintomáticos de recurrencia; no hay un sistema ideal pero parece claro que a mayor tiempo de monitorización, mayor probabilidad de diagnóstico. Un estudio japonés de 342 pacientes postablación, la mayoría paroxística $(60 \%)$, encontró recurrencias en 83 casos, de los cuales 60 (72,3\%) eran asintomáticos. El uso del Holter reveló solo $24 \%$ de las recaídas detectadas por el monitor externo ${ }^{17}$.

La habilidad diagnóstica de recurrencia de fibrilación auricular postablación de la telemetría ambulatoria se evaluó en un estudio de 300 pacientes, en quienes se ubicó el dispositivo durante 30 días y detectó recurrencias en 169 casos (53\%) demostrando así su utilidad. Esta reaparición de eventos temprana se asoció inversamente con la probabilidad de estar libre de arritmias a un año $(38,1 \%$ vs. $79,5 \% \mathrm{p}<0,001)$; de igual modo, la presencia de múltiples eventos predice de manera más contundente la falla del procedimiento a largo término (OR 4,5 IC95\% 2,3-8,8) ${ }^{18}$. Sin embargo, hay menos estudios con esta tecnología.

Finalmente, es importante reseñar que en la actualidad en el país no hay monitores externos de eventos con capacidad de autodetección de fibrilación auricular y que existe un dispositivo de parche de telemetría ambulatoria de Medtronic (Sistema SEEQ de telemetría móvil cardiaca).

Una limitante por definir es la relación costo-beneficio de estos dispositivos, no solo en cuanto al diagnóstico sino a la mejoría de la calidad de vida de los pacientes.

\section{Recomendaciones}

\section{Clase I}

- El monitor externo de eventos está indicado en el paciente con síntomas sugestivos de fibrilación auricular cuando la periodicidad de ellos sea al menos una vez al mes (nivel de evidencia $\mathrm{C}$ ).

- El monitor externo de eventos con autodetección de fibrilación auricular o la telemetría ambulatoria, están indicados en el estudio de los pacientes con eventos cerebro-vasculares o ataque isquémico transitorio criptogénico (que incluye estudio inicial con neuroimágenes, ecocardiograma transtorácico y transesofágico y estudios vasculares de cuello e intracraneanos negativos), cuando los estudios intrahospitalarios (ECG, monitorízación, telemetría o Holter) han sido negativos (nivel de evidencia B).

\section{Clase II a}

- El monitor externo de eventos que autodetecta fibrilación auricular puede ser útil para descubrir recaídas en pacientes sometidos a ablación de fibrilación auricular (nivel de evidencia C).

\section{Clase II b}

- La telemetría ambulatoria posiblemente es útil para la detección de recaídas en pacientes sometidos a ablación de fibrilación auricular (nivel de evidencia C).

\section{Conflicto de intereses}

Los autores declaran no tener conflicto de intereses.

\section{Bibliografía}

1. Mora G, Uribe W, Orjuela A, et al. Guías colombianas de electrofisiología no invasiva. Rev Colom Cardiol. 2014;21 Supl 1: $1-127$.

2. Rothman SA, Laughlin JC, Seltzer J, et al. The diagnosis of cardiac arrhythmias: a prospective multi-center randomized study comparing mobile cardiac outpatient telemetry versus standard loop event monitoring. J Cardiovasc Electrophysiol. 2007; 18:241-7.

3. Carvajal J, Clavijo C, Bautista L, Mora G. Caracteristicas clínicas de pacientes llevados a monitoria externa de eventos. Rev Colomb Cardiol. 2014;21:278-83.

4. Jauch F, Saber JL, Adams J, et al. Guidelines for the early management of patients with acute ischemic stroke a guideline for healthcare professionals from the American Heart Association/American Stroke Association. Stroke. 2013;44: 870-947.

5. Wolf PA, Abbot RD, Kannel WB. Atrial fibrillation as an independent risk factor for stroke: The Framingham study. Stroke. 1991;22:983-8.

6. Tayel AH, Tian KM, Kelly M, et al. Atrial fibrillation detected by mobile cardiac outpatient telemetry in cryptogenic TIA or stroke. Neurology. 2008;71:1697-701.

7. Hart RG, Diener HC, Coutts JB, et al., Cryptogenic Stroke ESUS International working group. Embolic strokes of undetermined source: the access for a new clinical construct. Lancet Neurol. 2014;13:429-38.

8. Gladstone D, Spring M, Dorian P, et al. Atrial fibrillation in patients with cryptogenic stroke. N Eng J Med. 2014;370:2467-77.

9. Sanna T, Diener HC, Passman RS, et al. Cryptogenic stroke and underlying atrial fibrillation. N Eng J Med. 2014;370: 2478-86.

10. Yayehd K, Irles D, Akret C, et al. Detection of paroxysmal atrial fibrillation by prolonged electrocardiographic recording after ischaemic stroke in patients aged $<60$ years: a study with 21 day recording using the Spider Flash monitor. Arch Cardiovasc Dis. 2015;108:189-96.

11. Miller DJ, Khan MA, Schultz UR, et al. Outpatient cardiac telemetry detects a high rate of atrial fibrillation in cryptogenic stroke. J Neurol Sci. 2013;324:57-61.

12. Favilla CG, Ingala E, Jara J. Predictors of finding occult atrial fibrillation after cryptogenic stroke. Stroke. 2015;46:120-5.

13. Kalani R, Bernestein R, Passman R, et al. Low yield of mobile cardiac outpatient telemetry after cryptogenic stroke in patients with extensive cardiac imaging. J Stroke Cerebrovasc Dis. 2015;24:2069-73.

14. Sposato L, Cipriano L, Saposnik G, et al. Diagnosis of atrial fibrillation after stroke and transient ischaemics attack: a systematic review and meta-analysis. Lancet Neurol. 2015;4:377-87.

15. Mittal S, Movsowitz C, Steinberg J. Ambulatory external electrocardiographic monitoring. J Am Coll Cardiol. 2011;58: 1741-9.

16. Joshi S, Choi AD, Kameth GS, et al. Prevalence, predictors, and prognosis of atrial fibrillation early pulmonary vein 
isolation: findings from 3 moths of continuous automatic ECG loop recording. J Cardiovasc Electrophysiol. 2009;20:1089-94.

17. Takagi T, Miyazaki S, Kusa S, et al. Role of extended external auto-triggered loop recorder monitoring for atrial fibrillation. Circ J. 2014;78:2637-42.
18. Liang JJ, Elafros MA, Chik WW, et al. Early recurrence of atrial arrhythmias following pulmonary vein atrial isolation. Timing and frequency of early recurrences predicts long-term ablation success. Heart Rhythm. 2015;12:2461-8. 